\title{
The word superiority effect: A comparison between restricted and unrestricted alternative set
}

\author{
AMOS SPECTOR and DEAN G. PURCELL \\ Oakland University, Rochester, Michigan 48063
}

\begin{abstract}
Three experiments were conducted to investigate the word superiority effect (WSE) (Reicher, 1969). The first two experiments used mixed presentations of words and nonwords, and positional uncertainty of the critical letter. Experiment 1 used an unrestricted set of alternatives, while Experiment 2 used only two alternatives ( $R$ and $L)$. Experiment 3 compared letter detection in nonwords with a restricted and unrestricted alternative set. WSE was found for both Experiments 1 and 2, at about the same level. Experiment 3 showed superior performance when alternatives were known in advance. It was concliuded that context has an effect on letter recognition even with prior knowledge of alternatives if the critical position is not known in advance. Some incompatibilities between the present results and those of other investigators in the field are discussed.
\end{abstract}

The word superiority effect (WSE) refers to a finding initially reported by Reicher (1969) and by Wheeler (1970): when a subject has to identify a letter, he does better when the letter is embedded within a word than when it is embedded in a nonsense string of letters or presented alone. As stated above, the effect is not surprising, since when presented with a word the subject might guess the identity of the letter using the redundancy inherent in English words. However, the Reicher experimental technique attempted to eliminate this problem. Rather than identifying the critical letter, Reicher's subjects had to choose between two alternative letters, one of which was the critical letter. The alternatives were such that if the presented stimulus was a word both alternatives would form a word. Thus, if the stimulus presented was the word "DOVE," the two alternatives for the first position letter might be " $D$ " and " $L$." Reicher reasoned that this would eliminate the differential advantage of guessing for word over nonword stimuli.

A similar finding was reported by Smith and Haviland (1972). Eliminating the advantage of redundancy by using the Reicher's forced choice technique, these authors found that letters embedded in words are chosen better than letters embedded in nonsense strings of letters, despite subject's knowledge of the rules generating the nonsense strings. Aderman and Smith (1971), in a related finding, showed that letters embedded in spelling patterns are perceived better than letters embedded in unrelated strings. They further showed that this advantage of the spelling pattern depends on the

This research was supported in part by an Oakland University research grant. Reprint requests should be sent to Amos Spector, Psychology Department, Oakland University, Rochester, Michigan 48063. subject's expectancy of being presented with a spelling pattern.

All these findings suggest the conclusion that a target letter embedded in a word context is perceived better than a target letter embedded in a nonword context. However, Massaro (1973) and Thompson and Massaro (1973) raised an objection to the above conclusion. They suggested that, at least when the critical letter is embedded in a word, the subject does not consider the alternatives while deciding on the critical letter. Thus, partial information extracted from the target letter would be matched against a set of possible alternatives that is reduced to those which would form a word. If this is the case, then the Reicher forced-choice technique does not eliminate the word advantage due to redundancy.

In order to test this possibility, Massaro (1973) and Thompson and Massaro (1973, Experiment II) extended the Reicher forced-choice technique by using a restricted set of alternatives. They argued that restricting the number of alternatives would sensitize the subject to their particular features and thus eliminate his tendency to reach a decision about the word prior to considering the alternatives. "Given sufficient practice with this fixed set of alternatives, $S$ should be able to incorporate the knowledge of the alternatives into the perceptual process eliminating the effects of redundancy" (Massaro, 1973, p. 350). Massaro (1973 and Thompson and Massaro (1973) failed to find the WSE. This result suggests that prior knowledge of the alternatives will eliminate the WSE. Smith and Haviland (1972) report WSE using a restricted set of alternatives (eight for words and eight for nonwords). Their subjects were informed about, and practiced with, the letter alternatives. However, Smith and Haviland's procedure was such that the response alternatives were presented to the subject outside the tachistoscope after each trial. 
As Massaro (1973) argues, this procedure might allow for differential forgetting of word and nonword stimuli, since words are easier to rehearse.

Johnston and McClelland (1974) pointed out a possible deficiency in Massaro's and Thompson and Massaro's experiments. Johnston and McClelland investigated the effects of subjects' strategy on WSE. They found that when the stimulus was a word, an instruction to look at the whole stimulus lead to better performance than an instruction to concentrate on the critical position. When the stimulus was a nonword, an opposite effect was observed. They suggest that Massaro and Thompson and Massaro (Experiment II) confounded the results of their studies where they failed to find WSE, since the critical letter in these studies always appeared in the second serial position. Their subjects probably focused only on the second serial position and lost any advantage that letters in words have over single letters or nonwords.

In a series of studies, Bjork and Estes (1973), Estes (1975), and Estes, Bjork, and Skaar (1974) failed to find evidence for WSE despite the use of random position of the critical letter. In all of the three studies, the subjects had prior knowledge of the alternative set (only $\mathrm{R}$ and $\mathrm{L}$ were used as the target letters), in addition to being unable to predict the critical position. In contrast to the above studies, Carr, Lehmkuhle, Kottas, Astor-Stetson, and Arnold (1976) found WSE with positional uncertainty of the critical letter, using a restricted set of alternatives. A possibly critical difference between the experiments of Carr et al. and those of Bjork and Estes (1973), Estes (1975), and Estes et al. (1974) is that the latter presented words and nonwords mixed within blocks of trials, whereas the former blocked the presentation by stimulus type. Carr et al. states that no WSE has been found, with the exception of Reicher (1969), where words and nonwords were mixed. They conclude that "knowing the target will be part of a word may be prerequisite to the word superiority effect."

Another possible reason for the failure on the part of Bjork and Estes (1973), Estes (1975) and Estes et al. (1974) to find the WSE could be their stimulus materials. Their stimuli were generated and presented in a way that might have minimized the differences between words and nonwords. Specifically: (a) They used a computer-controlled oscilloscope display in which each character was generated by illuminating the appropriate pattern of points on a 6 by 5 matrix; with this display there might have been a loss of definition for some features of individual letters as compared to typewritten letters. (b) The four letter displays subtended over $4^{\circ}$, which could cause a loss of acuity for features in positions other than those upon which the subject fixates. (c) The manner in which Bjork, Estes, and Skaar generated their stimuli might have caused their nonwords to be quite similar to words. Half of their nonwords were such that replacing the critical letter by the alternative would produce a word. Since $R$ and $L$ can replace each other in many positions relative to many letters in English, this procedure might lead to word-like strings. In many cases, these strings would be easily pronounceable and in some cases would be structured strictly of spelling patterns. In the examples SRED and TLAY, given by Bjork and Estes (1973), the initial SR and TL are not English spelling patterns. However, strings like BRUE and FOOR are very much English-like. The other half of the nonwords were generated from words by leaving the critical letter in its position, but rearranging the other letters, and keeping the original consonant vowel structure. This procedure leads to some clearly non-English-like strings, such as their example of YLAP and YRAP as generated from PLAY and PRAY. However, strings like DOLF and DORF (generated from FOLD and FORD) are easily pronounceable. Taking the above into consideration, it is possible that some of Bjork, Estes, and Skaar's nonwords were probably word-like. It is impossible to tell how many of their nonword s:imuli were wordlike without seeing the entire set.

To recapitulate, it is unclear whether prior knowledge of a restricted alternative set will eliminate WSE under conditions of positional uncertainty of the target letter and mixed presentation of words and nonwords. The experiments by Bjork, Estes, and Skaar suggest that WSE might be eliminated under these conditions, but because of the nature of their stimuli, their results may not be conclusive. The present experiments, therefore, employed mixed presentation of words and nonwords, and positional uncertainty of the target letter. The stimuli were conventional typed letters, presented tachistoscopically to the fovea. In addition, the alternatives were presented in the tachistoscope at the termination of the mask, so as to minimize or eliminate differential rehearsal or forgetting for words and nonwords. Experiment 1 replicated the procedure of Reicher (1969) in which the alternative set is unrestricted. Experiment 2 employed a restricted alternative set (the letters $R$ and $L$ ). Thus the experiments would allow for direct comparison of WSE with and without prior knowledge of the alternatives.

\section{EXPERIMENT 1}

The first experiment attempted to replicate the WSE and to provide data against which the results of Experiment 2 would be compared.

\section{Method}

Subjects. Ten undergraduate psychology students at Oakland University served as volunteer subjects. All had normal or corrected-to-normal vision. 
Stimulus materials. Seventy-two stimulus word pairs were chosen such that the two words differed on only one letter (e.g., DOVE-LOVE). Thus, one of the words of such a pair would be presented, followed by the two letter alternatives that would form the two different words if inserted into the critical position. The words were chosen such that the pair members would differ as little as possible on their frequency of usage. All words were composed of four letters, and the critical letter occupied each of the four possible positions equally often (18 times). The nonword stimuli were generated from the word stimuli by leaving the critical letter in its position and changing the positions of the other letters to form a nonsense string of letters. An attempt was made to make these strings unpronounceable. Each member of a nonword pair contained the same letters in the same order as the other member, except for the critical letter. Thus DOVE and LOVE could generate DVEO and LVEO as nonwords.

Two lists of stimuli were prepared, each to be presented to onehalf of the subjects. One member of each of the 72 word pairs went into each list. Similarly, one member of each of the 72 nonword pairs went into each list. The nonword that was assigned to one list was the one that contained the same critical letter as the word assigned to the same list. Thus, DOVE and DVEO would belong to list A, and LOVE and LVEO to list B. In all four of the above examples, the first letter was the critical one to be tested, with $D$ and $L$ as the two alternatives. Other words and nonwords tested critical letters at the other three positions. This procedure allowed a relatively clean assessment of WSE, since in both words and nonwords the same letter was tested in the same position in the presence of the same letters. Word frequency was considered when assigning the word pairs to the two lists. Of the pairs that did differ in frequency, half of the higher frequency words were assigned to list $\mathbf{A}$, the other half were assigned to list $\mathbf{B}$. This also held as closely as possible for each subset of 18 words whose critical letter occupied a given position. Thus, each of the two lists contained 144 items ( 72 words and 72 nonwords). The order of these stimuli was randomized, with the restrictions that (a) there be no run of more than three words or nonwords, and (b) each block of 18 trials contain nine words and nine nonwords.

The stimuli and alternatives were typed on $6 \times 9$ in. cards, using IBM Pica Prestige type. All were typed with uppercase letters. The stimuli were presented via an Iconix four-field tachistoscope, at a viewing distance of $88.9 \mathrm{~cm}$. Each four-letter stimulus subtended a visual angle of $.6^{\circ}$. The mask was made up of over-printed Xs and Os and was $.32^{\circ}$ high by $.94^{\circ}$ wide, covering the stimulus area. A single black fixation point was located in a separate field, just below the center of the stimulus field. The stimulus field was set at $63.66 \mathrm{~cd} / \mathrm{m}^{2}$, the mask field at $40.10 \mathrm{~cd} / \mathrm{m}^{2}$, and the continuously illuminated fixation field at $10.18 \mathrm{~cd} / \mathrm{m}^{2}$.

An alternative card was prepared for each stimulus. Each of these cards contained two alternative letters, one above and the other below the position of the critical letter. The other three positions were occupied by dashes (minus signs). Within each position tested and within each stimulus type (word or nonword), the correct alternative was above the position of the critical letter half the time and below that position half the time. The same alternative cards were used for both lists. Thus, if the top alternative was the correct one for a given stimulus in list $\mathrm{A}$, the bottom one would be the correct alternative for the corresponding stimulus in list B. Illumination of the alternative field was set at $63.66 \mathrm{~cd} / \mathrm{m}^{2}$.

In addition to the experimental stimuli, 104 practice stimuli were prepared, half of which were words and half nonwords. Only one practice list was prepared, and it was used for all subjects. Thus, each word pair contributed only one word item and one nonword item to the practice list. The purpose of the practice list was to give the subject practice in a tachistoscopic experiment and to assess his appropriate exposure conditions.

Procedure. The subjects were introduced to the tachistoscope and familiarized with it for about 20 trials in which they had to identify briefly exposed single letters. They were instructed to fixate at the fixation point. The stimulus appeared for $30 \mathrm{msec}$, followed by a variable blank interval (ISI) which was adjusted by the experimenter. The ISI was followed by a 100 -msec exposure of the mask. The ISI was adjusted such that subjects performed at roughly the $75 \%$ level.

Following this brief practice, the main experimental session began with 104 practice trials. Subjects were told that they would be presented with four-letter strings, some of which would be words and some of which would be nonwords. They were introduced to the alternative field and were instructed as to the positioning of the alternatives as specifying the serial position of the critical letter stimulus. Alternatives were presented at the termination of the mask and were exposed for about $3 \mathrm{sec}$, the exact duration depending on the ISI for the given subject. The subjects were instructed to guess when not sure which alternative was the critical letter.

Each trial was sequenced in the following way: The two experimenters correctly positioned the appropriate stimulus and alternatives cards; one experimenter gave a verbal "ready" signal; and the subject, when prepared, initiated with a finger switch the sequence of 30-msec stimulus, ISI, 100 -msec mask, and about $2.8 \mathrm{sec}$ of alternative field. A whole trial took $3 \mathrm{sec}$. The subject gave a verbal response which was recorded by one of the experimenters. This sequence was followed by about a 2-3-sec intertrial interval. During the 104 practice trials, the ISI was adjusted so as to yield about $75 \%$ correct report of the critical target letter.

The experimental trials proceeded in exactly the same fashion as the practice trials. The subjects were not informed that the first 104 trials were, in fact, practice trials. If during the experimental trials the subject's performance level shifted (as it occasionally did), the ISI was adjusted so as to keep the subject at about $\mathbf{7 5 \%}$ correct. Adjustments were made only on predetermined trials such that an equal number of words and nonwords were presented at a given ISI. Two short breaks were given to the subjects, one after about 85 practice trials and one at the midpoint of the 144 experimental trials. The whole session, including familiarization, practice, and experimental trials, took about $70 \mathrm{~min}$.

\section{Results}

For each subject, the percent correct decisions was computed for each of the two stimulus types (wordnonword) at each of the four-letter positions. Table 1 displays the mean percent correct decisions collapsed across subjects. These data were subjected to a fully within two-way analysis of variance, with stimulus type and position of the tested letter as the factors. The results of the analysis showed that letters embedded in words were reported better than those embedded in nonwords, $F(1,9)=11.73, p<.01$. The average correct responses for words and for nonwords were $73 \%$ and $65 \%$, respectively. Neither the position effect nor its interaction with stimulus type were significant.

\section{Discussion}

The results of Experiment 1 replicated the findings reported by Reicher (1969) and Wheeler (1970). The magnitude of the WSE found here (about $8 \%$ ) is in line with previous findings. This result was obtained despite the fact that the experimental trials were not blocked by stimulus type. Both words and nonwords were mixed in one experimental session. This fact is not consistent with the belief expressed by Carr et al. (1976) that WSE would be found only when the different stimulus types are presented in blocks. 
Table 1

Mean Percent Correct Decision for Words and Nonwords on the Four Letter Positions in Experiment 1

\begin{tabular}{lccccc}
\hline & \multicolumn{5}{c}{ Position } \\
\cline { 2 - 5 } & 1 & 2 & 3 & 4 \\
\hline Words & 74 & 72 & 75 & 71 \\
Nonwords & 69 & 67 & 59 & 63 \\
\hline
\end{tabular}

One final note: Despite the failure to find a Position by Stimulus Type interaction, examination of Table 1 indicates a stronger position effect on nonwords than on words. This point will be elaborated further when Experiment 2 is described.

\section{EXPERIMENT 2}

Experiment 2 was designed to test the existence and magnitude of the WSE under conditions where the stimulus set is unrestricted but the alternative set is minimal in size. As in Experiment 1, the position of the critical letter varied unpredictably between trials.

\section{Method}

Subjects. Ten undergraduate psychology students at Oakland University volunteered to serve as subjects. All had normal or corrected-to-normal vision. None of them had participated in Experiment 1.

Apparatus and materials. The stimulus materials were constructed in a similar way to those of Experiment 1, with the following differences: (a) The word pairs differed on only one letter, as in Experiment 1, but that letter was always $R$ in one word and $L$ in the other word. Thus, the alternative set was restricted to these two letters. (b) Having exhausted the English dictionary, it became obvious that it would be impossible to find enough of the four-letter word pairs to enable both words to be relatively common. This held true especially because it was decided to test each of the four positions an equal number of times (as in Experiment 1). Therefore, 36 word pairs ( 9 pairs for each critical position) were chosen. Lists $A$ and $B$ were generated from them, as in Experiment 1. Each list contained 36 words and 36 nonwords. Both lists were presented to each subject, with half receiving list $\mathbf{A}$ first and half receiving list $B$ first. Accordingly, each subject was presented with 144 experimental trials. (c) There were 104 practice trials, in all of which the alternatives were $R$ and L. Other than the above differences, the materials were prepared in a manner identical to that of Experiment 1, with control of the same factors (frequency in English, positioning of the correct letter on the alternative card, and sequencing of the stimuli). Luminance levels and exposure durations were the same as in Experiment 1.

Procedure. The procedure was the same as in Experiment 1, with the necessary changes in instructions to restrict the alternative set to $R$ and $L$.

\section{Results}

Each subject's data were transformed into percent correct decisions and broken down by stimulus type (word-nonword), position of critical letter, and correct response ( $\mathrm{R}$ or $\mathrm{L})$. These data were subjected to a three-way analysis of variance, with all factors being within-subject. The correct response factor ( $R$ vs. $L$ ) did not produce a significant main effect, nor did it interact with any of the other factors or their interaction. Thus, whether the critical letter was $R$ or $L$ did not seem to make any difference. The data were therefore collapsed across this factor. Table 2 shows the mean percent correct, collapsed across subjects, for the two stimulus types at the four serial positions. An analysis of variance performed on these data showed a significant WSE, $F(1,9)=$ $17.00, \mathrm{p}<.01$. The means for words and nonwords were $77 \%$ and $68 \%$, respectively. Both the position effect, $F(3,9)=4.11, p<.02$, and Position by Stimulus Type interaction, $F(3,9)=5.23, p<.01$, were significant. Table 2 shows that nonsense strings are affected by position, whereas words do not seem to be. This fact is similar to the nonsignificant finding of Experiment 1.

It can be argued that the finding of WSE with a restricted alternative set was due to the fact that each subject saw both members of each word pair, facilitating somehow his performance on the second member. If this were the case, then little or no WSE should be observed for the first half of the trials given to the subject. However, when the subjects' data from the first half of the trials were analyzed, the word advantage was even larger than it was for the whole experiment $(14 \%)$. Thus, the WSE observed in Experiment 2 is not an artifact of presenting both members of each stimulus word pair to each subject.

\section{Comparison Between Experiments 1 and 2}

The data from both experiments were subjected to a three-way analysis of variance, with stimulus type and position of critical letter as within-subject factors and experiment (1 vs. 2) as a between-subjects factor. The results of this analysis yielded, of course, a significant stimulus type effect, $F(1,18)=28.26$, $p<.001$. It also yielded a significant position effect, $F(3,54)=3,63, p<.02$, and a significant interaction of Position by Stimulus Type, $F(3,54)=3.74$, $\mathrm{p}<.02$. More importantly, however, the experiment factor did not interact with any of the other factors or with their interaction, the largest of these three $F$ ratios being 1.18. Thus, it seems that whether the alternative set was restricted or unrestricted made no difference with respect to the factors investigated in the present two experiments. A special mention should be made regarding the Position by Stimulus Type interaction. This interaction was significant

Table 2

Mean Percent Correct Decision for Words and Nonwords on the Four Letter Positions in Experiment 2

\begin{tabular}{lcccc}
\hline & \multicolumn{4}{c}{ Position } \\
\cline { 2 - 5 } & 1 & 2 & 3 & 4 \\
\hline Words & 79 & 72 & 82 & 76 \\
Nonwords & 80 & 64 & 61 & 66 \\
\hline
\end{tabular}


in Experiment 2, but failed to reach significance in Experiment 1. As noted earlier, however, the direction of the interaction was the same in both experiments. Indeed, the present analysis yielded an $F$ ratio of .51 for the Experiment by Stimulus Type by Position interaction.

\section{EXPERIMENT 3}

The data presented in Experiments 1 and 2 do not provide the information necessary for an overall comparison between performance levels in the two experiments. There are two reasons for this: (a) Overall performance levels differed slightly between the two experiments. Since the slope of ISl as a function of performance is unknown for the stimuli we used, we cannot correct for differences in performance. (b) For those subjects whose ISI was adjusted during the experimental sessions, it is not possible to decide which ISI to report (see method of Experiment 1). Therefore, Experiment 3 was designed to allow a direct comparison between performance levels under the two conditions investigated in this paper: prior knowledge and no prior knowledge of the alternatives.

\section{Method}

Subjects. Ten volunteer undergraduate psychology students at Oakland University participated in the experiment.

Stimulus materials. The nonword stimuli from Experiments 1 and 2 were used as stimuli. There was one block of 72 nonwords with $R$ and $L$ as alternatives, and one block of 72 nonwords with unrestricted alternatives. Each serial position was tested 18 times in each of the two blocks. Practice lists were composed of the 52 nonwords from the practice lists of Experiments 1 and 2.

Design and Procedure. Each subject received both blocks of experimental trials with a short break between them. Half the subjects got the R-L block first, the other half got it second. Prior to being tested on the first block, the subject was given the 52 practice trials pertaining to that block. During these initial practice trials, an ISI was determined which yielded roughly $75 \%$ performance. This ISI was fixed for the rest of the experiment. Prior to the second block of trials, the subject was given 15 practice trials corresponding to the condition investigated in that block. Prior to the practice trials for each block, the subjects received full instructions as to the nature of the task and the nature of the alternative set. The apparatus and viewing conditions were identical to those used in Experiments 1 and 2.

\section{Results}

The percent of correct detections for each of the two conditions and for each of the four positions of the critical letter was recorded for every subject. These data were subjected to a two-way analysis of variance, with alternative set and position tested as within-subject factors. The results of the analysis of variance showed a significant effect of alternative set, $F(1,9)=7.98, p<.02$. The subjects performed better when they had prior knowledge of the alternatives $(76.5 \%)$ than when they did not $(68.3 \%)$. The effect of position was also evident, $F(3,27)=20.76$, $p<.001$, replicating the position curve obtained for nonwords in the previous experiments: Performance rates for Positions 1 through 4 were $87 \%, 72 \%$, $61 \%$, and $64 \%$, respectively. The interaction of Prior Knowledge by Position was marginally significant, $F(3,27)=2.92, .05<p<.1$. The source of the interaction is the last position which shows a higher advantage for the restricted alternative set over the unrestricted set.

\section{GENERAL DISCUSSION}

The WSE was found with a minimal set of alternatives. Furthermore, this effect did not show any sign of being smaller than WSE when the alternative set was unrestricted (8\% in Experiment 1 as compared to $9 \%$ in Experiment 2). It seems, therefore, that just prior knowledge of the alternatives and practice with them does not eliminate the WSE. Thus the present findings are not consistent with the findings of Bjork and Estes (1973), Estes (1975), and Estes et al. (1974). It seems likely that the source of this inconsistency lies in the difference between the two sets of experiments with respect to stimulus materials and display type, as described in the introduction. The results of Experiment 3 show that the observer benefits from a prior knowledge of the alternatives, apparently by sensitizing himself to their particular features. Taken together with the results of Experiments 1 and 2, which show no Prior Knowledge by Stimulus Type interaction, the present findings are in disagreement with Massaro's (1973) claim that WSE will be eliminated with prior knowledge of the alternative set. Massaro's (1973) failure to find WSE is probably due to his using the second position of a three-letter stimulus as a fixed critical position. Johnston and McClelland (1974) demonstrated that performance on critical letters embedded in words decreased when the subjects were encouraged to concentrate on the tested position. In addition, Carr et al. (1976) showed that the middle position of a three-letter display produced the weakest WSE. It is possible that both of these factors were operating in Massaro's experiment. Furthermore, his three-letter stimulus subtended over $3^{\circ}$ and, because of its size, might have encouraged subjects to concentrate only on the middle position. It is, therefore, probable that Massaro's failure to observe WSE was not entirely due to his subjects' prior knowledge of the alternatives.

The results of the present experiments support the notion that the detection of a letter is affected by the context in which it appears. Not only is detection of a letter in a word superior to detection of a letter in a nonword, but the serial position functions are also different. Experiments 1 and 2 suggest a relatively flat position function for word context. All three experiments indicate a U-shaped position function for nonwords. It seems, therefore, that the processes 
determining letter detection in a word are different from those determining letter detection in a nonsense letter string. These processes, as shown by other investigators, depend upon the observer's strategy (Aderman \& Smith, 1971; Johnston \& McClelland, 1974). Aderman and Smith (1971) showed that a letter embedded in a spelling pattern is identified better than a letter embedded in a nonsense string, and that this holds only if the subject expects a spelling pattern. This finding might explain why Carr et al. (1976) claim that a necessary condition to obtain the WSE is to present stimulus type by blocks. However, the results of the present experiments show that blocking the stimulus type is not necessary. This is not contradictory to the Aderman and Smith finding. It only suggests that, on a continuum ranging from "expecting a word" to "expecting a nonword," one should push quite far toward expectancy of a nonword in order to eliminate WSE. Thus, Aderman and Smith's subjects expected a nonword, whereas the subjects in the present experiment knew that they were going to be presented with either a word or a nonword. Apparently the subjects prefer to use that perceptual strategy which, from the experimenter's point of view, will result in WSE when there is the possibility of a word being presented.

The Position by Stimulus Type interaction reported here is not in agreement with the interaction found by Carr et al. (1976). Carr et al. found a U-shape position effect for words and an inverse $U$ for nonwords. The present experiment found a relatively flat function for words and a U-shaped function for nonwords. Two points relate to this discrepancy: (a) The two experiments are not directly comparable with respect to position functions, since Carr et al. used three-letter stimuli subtending $1.0^{\circ}$ of visual angle and the present experiment used fourletter stimuli subtending $0.6^{\circ}$. Thus, their spacing between the letters was larger than ours. (b) The $\mathrm{U}$ function reported by Carr et al. for words does not appear to be very steep and might not be different from a flat curve.
In conclusion, the present study presents data which support the original Reicher (1969) finding of WSE with mixed presentation of words and nonwords. Restricting the alternatives set did not result in any reliable change in the magnitude of this effect. It seems that under conditions of prior knowledge with positional uncertainty WSE is not eliminated, even with mixed presentation of words and nonwords.

\section{REFERENCES}

Aderman, D., \& Smith, E. E. Expectancy as a determinant of functional units in perceptual recognition. Cognitive Psychology, 1971, 2, 117-129.

BjoRK, E. L., \& Estes, W. K. Letter identification in relation to lingu istic context and masking conditions. Memory \& Cognition, $1973,1,217-223$.

Carr, T. H., Lehmkuhle, S. W., Kottas, B., Astor-Strtson, E. C., \& ARnold, D. Target position and practice in the identification of letters in varying contexts: A word superiority effect. Perception \& Psychophysics, 1976, 19, 412-416.

EsTEs, W. K. The locus of inferential and perceptual processes in letter identification. Joumal of Experimental Psychology: General, 1975, 104, 122-145.

Estes, W. K., Bjork, E. L., \& SKaAR, E. Detection of single letters and letters in words with changing vs. unchanging mask characters. Bulletin of the Psychonomic Society, 1974, 3, 201-203.

Johnston, J. C., \& McClelland, J. L. Perception of letters in words: Seek not and ye shall find. Science, 1974, 184, 1192-1194.

Massaro, D. W. Perception of letters, words, and nonwords. Journal of Experimental Psychology, 1973, 100, 349-353.

REICHER, G. M. Perceptual recognition as a function of meaningfulness of stimulus materials. Journal of Experimental Psychology, 1969, 81, 275-280.

Smith, E. E., \& Haviland, S. E. Why words are perceived more accurately than nonwords: Inference vs. unitization. Journal of Experimental Psychology, 1972, 92, 59-64.

Thompson, M. C., \& Massaro, D. W. Visual information and redundancy in reading. Journal of Experimental Psychology, $1973,98,49-54$.

WheEler, D. D. Processes in word recognition. Cognitive Psychology, 1970, 1, 59-85.

(Received for publication September 27, 1976; revision accepted December 27, 1976.) 